\title{
Development and Proof-of-Concept Study of a Novel Intraoperative Surgical Planning Tool for Robotic Arm-Assisted Total Knee Arthroplasty
}

\author{
Daniel Farley*, Gladius Lewis \\ Department of Mechanical Engineering, The University of Memphis, Memphis, TN, USA \\ Email: ^dannypfarley@gmail.com
}

How to cite this paper: Farley, D. and Lewis, G. (2020) Development and Proofof-Concept Study of a Novel Intraoperative Surgical Planning Tool for Robotic ArmAssisted Total Knee Arthroplasty. World Journal of Engineering and Technology, 8, 505-522.

https://doi.org/10.4236/wjet.2020.83036

Received: July 15, 2020

Accepted: August 23, 2020

Published: August 26, 2020

Copyright () 2020 by author(s) and Scientific Research Publishing Inc. This work is licensed under the Creative Commons Attribution International License (CC BY 4.0).

http://creativecommons.org/licenses/by/4.0/

\section{(c) (i) Open Access}

\begin{abstract}
Background: Intraoperative surgical planning tools (ISPTs) used in current-generation robotic arm-assisted total knee arthroplasty (RTKA) systems (such as Navio and $\mathrm{MAKO}^{\circ}$ ) involve employment of postoperative passive joint balancing. This results in improper ligament tension, which may negatively impact joint stability, which, in turn, may adversely affect patient function after TKA. Methods: A simulation-enhanced ISPT (SEISPT) that provides insights relating to postoperative active joint mechanics was developed. This involved four steps: 1) validation of a multi-body musculoskeletal model; 2) optimization of the validated model; 3) use of the validated and optimized model to derive knee performance equations (KPEs), which are equations that relate implant component characteristics to implant component biomechanical responses; and 4) optimization of the KPEs with respect to these responses. In a proof-of-concept study, KPEs that involved two component biomechanical responses that have been shown to strongly correlate with poor proprioception (a common patient complaint post-TKA) were used to calculate optimal positions and orientations of the femoral and tibial components in the TKA design implanted in one subject (as reported in a publicly-available dataset). Results: The differences between the calculated implant positions and orientations and the corresponding achieved values for the implant components in the subject were not similar to component position and orientation errors reported in biomechanical literature studies involving Navio and MAKO ${ }^{\circ}$. Also, we indicate how SEISPT could be incorporated into the surgical workflow of Navio with minimal disruption and increase in cost. Conclusion: SEISPT is a plausible alternative to current-generation ISPTs.
\end{abstract}




\section{Keywords}

Robotic Arm-Assisted Total Knee Arthroplasty, Multi-Body Musculoskeletal Simulation Model, Knee Biomechanics, Rigid-Body Dynamics

\section{Introduction}

Total knee arthroplasty (TKA) is the most widely used surgical option for treatment/management of end-stage osteoarthritis [1] [2]. In some TKA series, patient dissatisfaction is up to 20\% [3] [4], with common complaints being anterior knee pain and compromised proprioception [5] [6] [7] [8]. To address this issue, several developments have been introduced, among which is robotic arm-assisted TKA (RTKA) [9]-[14]. Available clinical evidence shows that when RTKA is used, there is precision of bone preparation, improved accuracy of alignment and positioning of implant components, marked reduction in the number of outliers in component position and alignment, and reduced intraoperative malalignment of components [9] [10] [15]. Additionally, compared to surgery performed using conventional instrumentation (CI), benefits of RTKA include reduced time-to-hospital discharge and decreased episode-of-care cost [16] [17] [18]. On the other hand, RTKA has risks, such as loosening or fracture of pin trackers; complications, such as pin site infections; and shortcomings, such as additional radiation exposure when an image-based platform is used [9]. Additionally, unequivocal data on some aspects of RTKA are not currently available, examples being operative time, learning curve (especially for low-TKA-volume surgeons), and cost-benefit ratio [9] [11].

In most series, significant increases in various patient outcome measures or patient satisfaction in an RTKA group, relative to the corresponding value in the CI surgery group, have not been reported [19] [20]. One contributing factor to this record is that in both types of surgeries, gap balancing is done in a passive state; that is, it is done while the patient is on the operating table under anesthesia [12]. In other words, the influence of active soft tissue forces, and, even, of gravity are not considered, which is likely to adversely affect the patient's functional stability after the arthroplasty [21]. Current technologies that aim to improve soft-tissue tensioning and balancing have shortcomings: with computer-assisted gap balancing systems and intraoperative use of tibial insert sensors [22], there is no kinetic feedback because they are used while the patient is in a non-weight-bearing state under anesthesia; and, with a robotic-assisted active soft-tissue balancing system, there is, as yet, only very limited evaluation (one report of a cadaver study [23] and one report of early clinical results) [13]). Through a volitional flexion-extension maneuver test at the conclusion of a CI TKA, a well-balanced knee experiences an increase of between $\sim 200 \%$ and $\sim 300 \%$ in load across the joint under isometric muscular contraction [24]. While it is possible to characterize active joint biomechanics using techniques such as 
mobile fluoroscopy and radiostereometric analysis, they are time-consuming, expensive, and expose the patient to radiation [25].

An intraoperative surgical planning tool, which is an integral part of a RTKA system, allows the surgeon to utilize information about the patient's soft tissues to obtain a properly balanced joint replacement. In the process, the knee joint of the patient is flexed intraoperatively and the resulting kinematics data are used to create a model of the ligaments and tendons. These models provide the surgeon with patient-specific soft tissue data with which to plan the surgery and, as such, are very important. In spite of that, literature studies on intraoperative surgical planning tools for RTKA systems are lacking. There were three purposes of the present study. First, to develop a simulation-enhanced intraoperative surgical planning tool (SEISPT) for an RTKA system that can be used to provide the surgeon intraoperative feedback relating to the influence of active soft tissue behaviors on the operative plan. Second, to demonstrate the plausibility of SEISPT. Third, to examine the potential for SEISPT to be used with a current-generation RTKA system.

\section{Methods}

\subsection{Overview}

SEISPT was developed using results from analysis of a multi-body musculoskeletal model that includes a TKA design [26].

Four steps were involved (Figure 1). The first was validation of a multi-body musculoskeletal model using knee joint loading data obtained from subjects while they performed a common functional activity, namely, level walking. These data are contained in publicly-available benchmark datasets (OrthoLoad dataset [27] and 2014 SimTK dataset [28]). Second, design-of-experiments (DOE) methodology was used to optimize the validated model. Third, the validated and optimized model, together with the results of DOE studies that characterized the influence of a number of implant component variables on various biomechanical responses of the implant component and results of linear regression analysis, were used to develop a series of equations designated "knee performance equations" (KPEs). These equations give the relationship between model inputs (characteristics of an implant component) and outputs that, in literature studies, have been shown to be strongly and directly correlated with patient outcomes or complaints post-TKA. These outputs are designated "clinically-relevant biomechanical responses" (CRBRs). In the fourth step, the KPEs were used for optimization with respect to two CRBRs. The proof-of-concept study involved using the derived KPEs to compute the optimal position and orientation of the femoral and tibial components of the TKA design implanted in one subject (reported in 2014 SimTK dataset [28]).

\subsection{Validation of Multi-Body Musculoskeletal Model}

The multi-body musculoskeletal model used was one that has detailed soft tissue 


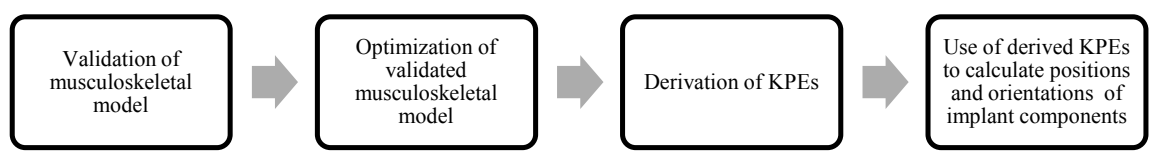

Figure 1. A schema of the four steps in the development of a simulation-enhanced intraoperative surgical planning tool for a robotic arm-assisted TKA system.

features surrounding the knee joint (VCTK-Knee; LifeModeler, Inc., San Clemente, CA, USA). This model can be tailored to patient-specific dimensions utilizing custom scaling (LifeModeler") [29]. The annual "Grand Challenge to Predict In Vivo Knee Loads" Competition was first held in 2009 and the latest one was held in 2014 [30] [31]. Knee joint loading results used in these competitions are available in public datasets. As VCTK-Knee was originally developed for the left knee, subject data from the 2014 Competition (hereafter, this dataset is referred to as "2014 SimTK dataset") provided the most recently available data for a subject with a left-knee TKA component. As such, in the present study, this dataset was used to validate VCTK-Knee; specifically, data obtained from Subject PS (male; $1.80 \mathrm{~m}$ tall, $75 \mathrm{~kg}$ ) were used [28]. This dataset contained joint loading data obtained from the subject in whom a force-sensing telemetric tibial implant component of a TKA had been implanted; specifically, the component was equipped with a device comprising four load-sensing elements that measured axial load, anteroposterior moments, mediolateral moments, and shear forces [32]. The height and weight of Subject PS were used to scale patient-specific VCTK-Knee. Subject anatomy was further refined by adjusting individual bone segment lengths based on measurements from the subject's computed tomography (CT) data. VCTK-Knee allowed importation of the gait analysis marker data as motion agent elements. To account for misalignment between real and virtual markers, motion agents were attached to body segments (bones) with virtual spring elements, whose stiffness parameters were adjusted to achieve the desired kinematics.

Although results for a large number of joint parameters were provided to the Grand Challenge Competition participants, these were not available to workers, such as the present ones, who were not competitors; as such, in the present study, only joint contact forces and moments were used for model validation. It is to be noted that the Grand Challenge Competition was designed to be a blinded study, and, as such, gait trials were not accompanied by joint contact loading data. However, several other calibration trials with joint contact force data and associated kinematics and ground reaction force data were provided. Thus, in the present work, in a bid to identify loading pattern similarities, all calibration movement data from Subject PS were compared with gait joint contact force data contained in another publicly available dataset in which an instrumented tibial component of a TKA design was implanted in subjects after which they performed a variety of activities, among which was level walking (OrthoLoad dataset [27]). Through this method, the functional movement performed by Subject PS that was similar to normal-gait joint load by the Orthoload subjects 
was identified. Hereafter, this movement is referred to as the "equivalent functional movement".

\subsection{Optimization of Validated VCTK Model}

Preliminary analysis of baseline VCTK-Knee yielded predicted tibiofemoral joint (TFJ) contact force magnitude with a mean root-mean-square-error (RMSE) of $130 \%$ body weight (BW), which was markedly larger than that obtained by the 2014 Grand Competition winner for Patient PS (mean RMSE $=30 \%$ BW) in the normal walking trial simulation [33]. As such, DOE methodology was used to optimize the validated VCTK-Knee. Optimal implant component position and orientation and soft tissue properties were selected using optimization tools in a commercially-available software package (Adams/Insight ${ }^{\mathrm{TM}}$; MSC Corp., Santa Ana, CA, USA), with the optimization goal being minimization of RMSE between predicted and measured TFJ force for Subject PS. RMSE was chosen as the metric of optimization because it was used as the basis for deciding the winners of each of the Grand Challenge Competitions [30] [31] [33].

\subsection{Development of Knee Performance Equations}

Development of these equations was done using DOE techniques, a decision that was guided by the need to use only input factors that could be incorporated into a surgical planning tool for a current-generation RTKA system. In the development of the KPEs, the criterion used to select the output/response was that each output/response is related to factors that have been cited in the literature as those that strongly correlated with TKA patient satisfaction scores. To provide an advantage over surgical planning tools used in current-generation RTKA systems, the output/response factor list was further scrutinized to include only factors that could be influenced by active soft tissue loading. One factor to which patient dissatisfaction with TKA has been linked is poor proprioception [8]. The KPEs developed in the present study focused on outputs/responses that could have a possible effect on proprioception, leading to 11 input factors and 9 outcomes/responses (Table 1 and Table 2).

\subsection{Multi-Objective Optimization}

A fractional factorial experimental design was devised for the KPE DOE. After solving for each of the simulation runs, linear regression was used to develop a KPE for each output/response. This set of KPEs was utilized for multi-objective optimization using Adams/Insight ${ }^{\mathrm{TM}}$ to determine optimal implant component position and orientation for which mid-flexion instability parameters (which have been directly associated with proprioception [34] [35]) are minimized.

\subsection{Proof-of-Concept Study}

The essence of this study was to compare the implant component positions and orientations of the femoral and tibial components of the TKA design that was 
Table 1. Input factors and deviations of their values for the knee performance equations.

\begin{tabular}{cc}
\hline Knee performance equation input factor & $\begin{array}{c}\text { Deviation from optimized implant component } \\
\text { position and orientation pose }\end{array}$ \\
\hline Femoral component ML position & $\pm 5 \mathrm{~mm}$ \\
Femoral component AP position & $\pm 5 \mathrm{~mm}$ \\
Femoral component SI position & $\pm 5 \mathrm{~mm}$ \\
Tibial component ML position & $\pm 5 \mathrm{~mm}$ \\
Tibial component AP position & $\pm 5 \mathrm{~mm}$ \\
Tibial component SI position & $\pm 5 \mathrm{~mm}$ \\
Femoral component varus/valgus & \pm 0.05 radian \\
rotation & \pm 0.05 radian \\
Femoral compont internal/external & \\
rotation & \pm 0.05 radian \\
rotation & \pm 0.05 radian \\
Tibial component varus/valgus & \pm 0.05 radian \\
\hline
\end{tabular}

ML, medial-lateral; AP, anteroposterior; SI, superior-inferior.

Table 2. Influences on the outputs/responses of the knee performance equations.

\begin{tabular}{cc}
\hline Knee performance equation output/response & Target influence \\
\hline Anterior MCL strain & Mid-flexion stability [34] [35] \\
Posterior MCL strain & Mid-flexion stability [34] [35] \\
Anterior LCL strain & Mid-flexion stability [34] [35] \\
Posterior LCL strain & Mid-flexion stability [34] [35] \\
Quadriceps angle & Anterior knee pain [5] \\
Quadriceps force & Anterior knee pain [6] \\
Patellofemoral contact force & Anterior knee pain [7] \\
Tibial implant force & Implant longevity [36] \\
Tibiofemoral joint contact force & Joint balance [37] \\
\hline
\end{tabular}

MCL, medial collateral ligament; LCL, lateral collateral ligament.

implanted in Subject PS using CI surgery ("achieved passive surgical value") to corresponding values calculated using SEISPT ("conceptual active surgical plan value"). The positions and orientations of the femoral and tibial components were provided for Subject PS in the 2014 SimTK dataset; as such, these measurements were used as the achieved passive surgical values. Optimization of the KPEs was used to determine the positions and orientations of the femoral and tibial implant components of a TKA design that minimize the MCL and LCL strains to clinically normal levels, which were then used as the conceptual active surgical plan values. An additional optimization objective, which was to match the TFJ contact force results provided in the 2014 SimTK dataset, was imposed. 
This ensured that use of the conceptual active surgical plan resulted in physiologically-relevant loading and clinically-acceptable implant component positions and orientations.

To compare final implant component positions and orientations, a shared coordinate reference frame was created for the bone and the implant component models utilizing a commercially-available software package (Geomagic Studio; 3D Systems, Rock Hill, SC, USA). Bone and implant component models for Patient PS (2014 SimTK) were imported into Geomagic Studio and, then, a custom-written Matlab script was used to determine the positional transform between the bone and implant component models. Likewise, bone and implant component models were exported from the musculoskeletal model (optimized VCTK-Knee). The KPE was used to position the implant component relative to the bone in optimized VCTK-Knee. Geomagic Studio was also used to examine the difference between 1) the achieved passive surgical value for the position of an implant component and the corresponding conceptual active surgical plan value; and 2) the achieved passive surgical value for the orientation of an implant component and the corresponding conceptual active surgical plan value.

\section{Results}

Although the time scales for the single-leg stance by Subject PS (2014 SimTK dataset) and a single-gait phase by the subjects in the OrthoLoad dataset are different, the two load curves are qualitatively similar (Figure 2). Therefore, simulation models constructed with optimized VCTK-Knee using single-leg stance kinematics and ground force data for Patient PS are plausible.

Comparisons of the TFJ joint force and moment predicted using optimized VCTK-Knee and experimentally obtained for Subject PS during single-leg stance show that during the early part of the cycle $(0.3 \mathrm{~s}-0.8 \mathrm{~s})$, the two sets of force results are very close but, after that, the model either underestimates or overestimates the experimentally-obtained results (Figure 3). RMSE for TFJ contact load was substantially reduced when optimized VCTK-Knee was used, from $130 \%$ BW to $84 \%$ BW. RMSE for TFJ contact moment was substantially reduced when optimized VCTK-Knee was used, from $13.11 \mathrm{Nm}$ to $7.63 \mathrm{Nm}$. For single-leg stance movement, the most highly correlated KPEs using optimized VCTK-Knee were those for quadriceps force (Pearson's product-moment correlation coefficient $\left.\left(R^{2}\right)=0.85\right)$, medial compartment rollback $\left(R^{2}=0.79\right)$, lateral compartment rollback $\left(R^{2}=0.79\right)$, Bundle $2 \mathrm{LCL}$ strain $\left(\mathrm{R}^{2}=0.79\right)$, and Bundle 3 MCL strain $\left(\mathrm{R}^{2}=0.76\right)$.

In the proof-of-concept study, the determined optimal positions and orientations of the femoral and tibial components of the TKA design implanted in Subject PS ("conceptual active surgical plan values") are given in Table 3, together with the actual positions and orientations of the implant components in the TKA implanted in that subject (as reported in 2014 SimTK dataset) ("achieved passive surgical values"). 


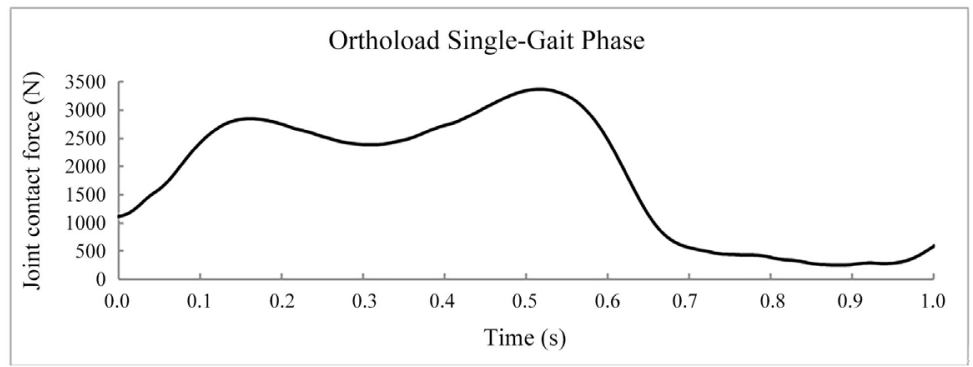

(a)

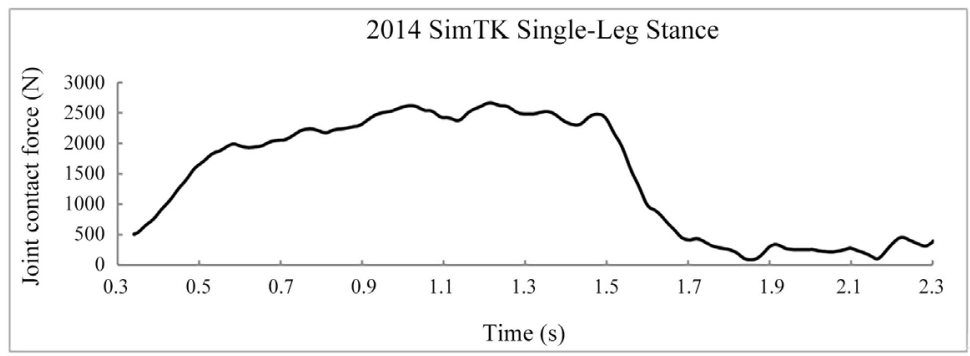

(b)

Figure 2. Comparison of tibiofemoral joint contact force in OrthoLoad dataset (single-gait phase) [27] (a) and in 2014 SimTK dataset (single-leg stance) (Subject PS) [28] (b).

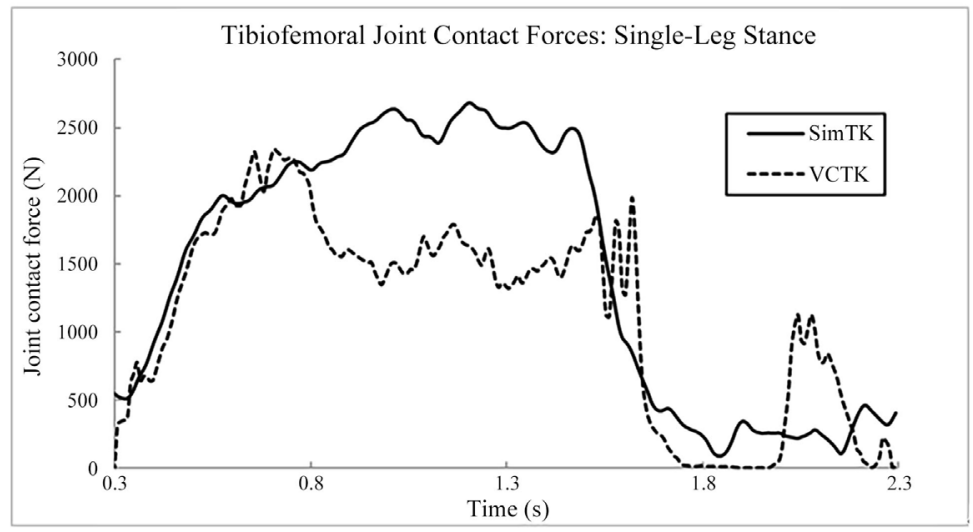

(a)

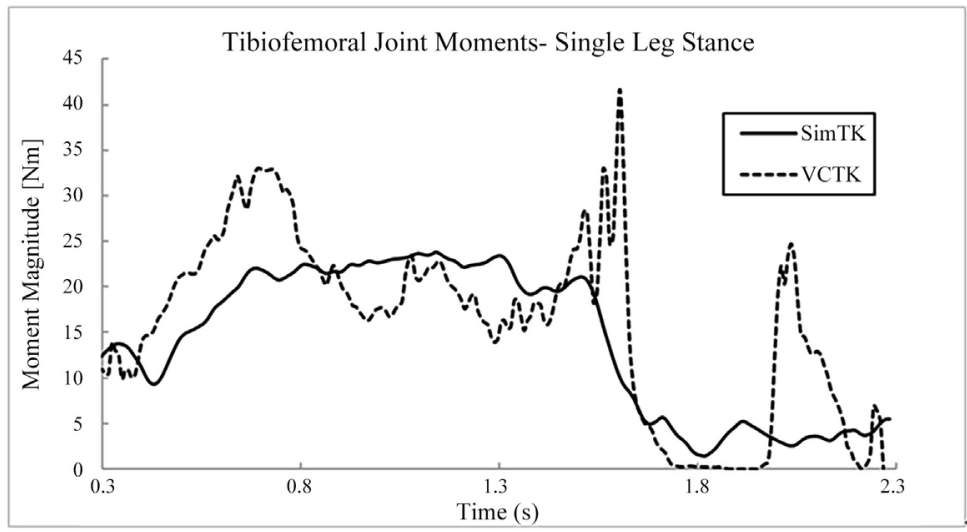

(b)

Figure 3. Variation with time of tibiofemoral joint contact force (a) and moment (b) for single-leg stance: optimized VCTK-Knee simulation model results versus 2014 SimTK dataset results (Subject PS) [28]. 
Table 3. TKA component position and orientation data comparing results achieved for Subject PS using a conventional instrumentation surgical method (gray) and results calculated for Subject PS using output from application of the conceptual active surgical plan (SEISPT) (blue). For comparison, component position and orientation errors reported by Jaramaz et al. [38] and by Hampp et al. [39] are included.

\begin{tabular}{|c|c|c|c|}
\hline $\begin{array}{l}\text { Femoral } \\
\text { component } \\
\text { position and } \\
\text { orientation }\end{array}$ & $\begin{array}{l}\text { Difference (achieved } \\
\text { using conventional } \\
\text { instrumentation surgery vs. } \\
\text { calculated using conceptual } \\
\text { active surgical plan (SEISPT) }\end{array}$ & $\begin{array}{l}\text { RMSE } \\
\text { with Navio } \\
\text { Surgical } \\
\text { System } \\
{[38]}\end{array}$ & $\begin{array}{l}\text { Robotic implant } \\
\text { alignment } \\
\text { accuracy error } \\
\text { (median } \pm \text { std. } \\
\text { dev.) [39] }\end{array}$ \\
\hline Medial-lateral & $4.23 \mathrm{~mm}$ & NS & NS \\
\hline Anterior-posterior & $\mathrm{r} 4.10 \mathrm{~mm}$ & $1.46 \mathrm{~mm}$ & NS \\
\hline Superior-inferior & $6.73 \mathrm{~mm}$ & $0.86 \mathrm{~mm}$ & NS \\
\hline Flexion-extension & $0.18^{\circ}$ & $2.18^{\circ}$ & $0.6^{\circ} \pm 0.5^{\circ}$ \\
\hline Varus-valgus & $3.73^{\circ}$ & $0.71^{\circ}$ & $0.6^{\circ} \pm 0.3^{\circ}$ \\
\hline Internal-external & $3.98^{\circ}$ & $0.69^{\circ}$ & $0.8^{\circ} \pm 0.5^{\circ}$ \\
\hline $\begin{array}{l}\text { Tibial component } \\
\text { position and } \\
\text { orientation }\end{array}$ & $\begin{array}{l}\text { Difference (achieved using } \\
\text { conventional } \\
\text { instrumentation surgery vs. } \\
\text { calculated using conceptual } \\
\text { active surgical plan (SEISPT) }\end{array}$ & $\begin{array}{l}\text { RMSE with } \\
\text { Navio } \\
\text { Surgical } \\
\text { System } \\
{[38]}\end{array}$ & $\begin{array}{l}\text { Robotic } \\
\text { implant alignment } \\
\text { accuracy error } \\
\text { (median } \pm \text { std. } \\
\text { dev.) [39] }\end{array}$ \\
\hline Medial-lateral & $3.68 \mathrm{~mm}$ & $1.29 \mathrm{~mm}$ & NS \\
\hline Anterior-posterior & $\mathrm{r} 1.26 \mathrm{~mm}$ & $0.79 \mathrm{~mm}$ & NS \\
\hline Superior-inferior & $7.60 \mathrm{~mm}$ & $0.68 \mathrm{~mm}$ & NS \\
\hline Flexion-extension & $1.58^{\circ}$ & $0.88^{\circ}$ & $1.1^{\circ} \pm 1.6^{\circ}$ \\
\hline Varus-valgus & $2.45^{\circ}$ & $0.69^{\circ}$ & $0.9^{\circ} \pm 0.4^{\circ}$ \\
\hline Internal-external & $3.20^{\circ}$ & $2.21^{\circ}$ & NS \\
\hline
\end{tabular}

Conventional instrumentation surgery: standard mid-vastus approach [40]. Root mean squared error $($ RMSE $)=($ component position/orientation achieved using Navio-component position/orientation planned using Navio). NS, result not stated in the report. Accuracy error = (component position/orientation planned using $\mathrm{MAKO}^{\circ}$ - component position/orientation planned using $\mathrm{MAKO}^{\circ}$ ).

\section{Discussion}

\section{Performance of Musculoskeletal Model}

Using optimized VCTK-Knee, predicted TFJ contact force was higher than that obtained by the winner of the 2014 Grand Challenge Competition (using 2014 SimTK dataset [28]) (mean RMSE $=84 \%$ BW versus 30\% BW [28]). This discrepancy could be attributed to four issues. First, VCTK-Knee was developed by utilizing a scaled skeletal model, instead of the exact bone anatomies provided in the 2014 SimTK dataset. This approach was necessary for this application so that body measurements (height, weight, and limb length) could be parameterized and included in embodiments of the KPEs. Compared to CT-derived models, the scaled femur and tibia models were $35 \mathrm{~mm}$ and $19 \mathrm{~mm}$ undersized, respectively. Improper scaling of the bone models could have resulted in inaccurate placement of the gait marker relative to the patient bone model. When 
musculoskeletal simulation models are used, marker position accuracy exerts a strong influence on the accuracy of the simulations [41]. Although, in the software package employed, use of motion agents offsets the effect of gait marker inaccuracies, the magnitude of this impact was not quantified. Second, an equivalent functional movement, rather than an actual experimentally-obtained functional movement, was used. Third, there is some uncertainty in some of the subject-specific model parameters, a perennial challenge with musculoskeletal modeling [42]. Fourth, there is some uncertainty about how the optimization criteria, contained in the software package employed, were used to predict the muscle activation pattern. Notwithstanding the aforementioned discrepancy, it is worth pointing out that the TFJ joint contact loading profiles obtained using optimized VCTK-Knee were qualitatively similar to those in the 2014 SimTK dataset $\left(\mathrm{R}^{2}\right.$ for force magnitude and moment magnitude $=0.84$ and 0.69 , respectively).

\section{Performance of SEISPT}

The literature on errors in component position/orientation when an RTKA system is used (error being defined as difference in component position/orientation achieved with an RTKA system and component position/orientation planned with the RTKA system) is very sparse [38] [39]. By this definition, the present results given in Table 3 (that is, difference between achieved component position/orientation using CI surgery and component position/orientation calculated using SEISPT) are not RTKA system errors, but, rather, notional errors. Nonetheless, it is instructive to note that these notional errors are noticeably different from 1) the RTKA system errors reported by Jaramaz et al. [38]; and 2) the means and standard deviations reported by Hampp et al. [39] (Table 3). This statement is made in full awareness of the fact that neither of these two referenced studies was a clinical investigation; specifically, in the Jaramaz et al. study [38], Navio ${ }^{\circledR}$ was used in the implantation of a state-of-the-art TKA design (Journey II XR; Smith \& Nephew, Memphis, TN, USA) in cadaver knees and synthetic bone specimens and, in the Hampp et al. study [39], MAKO ${ }^{\circ}$ was used in the implantation of an unspecified TKA design in cadaver knees. Nonetheless, when all is considered, the present results (Table 3) suggest that SEISPT may be a plausible alternative planning tool in the sense that the implant component position and orientation obtained by using it are different from the errors reported in the aforementioned biomechanical studies in which the two mostwidely used current-generation RTKA systems were used.

\section{Feasibility of SEISPT}

The feasibility of using SEISPT as part of an RTKA system is discussed from three perspectives. It is worth noting that current-generation RTKA systems feature either preoperative planning or intraoperative planning. With intraoperative planning, the surgeon characterizes the anatomy of the patient while he/she is on the operating table. This imposes a time constraint on the planning because, among other considerations, the risk for surgery-acquired infection increases with increase of time on the operating table [43]. 
The first perspective is that if SEISPT is to be considered for use in intraoperative surgery planning, it must be time-efficient; that is, the time to deploy it should be comparable to or, better still, shorter than time spent when intraoperative surgical planning tools used in current-generation RTKA systems are employed. The key to the time-efficiency of SEISPT is the optimization procedure that culminates in the generation of the KPEs, which, in turn, are used to compute a series of model output responses. DOE and linear regression allowed for the characterization of hundreds of simulation runs in a single linear equation. Optimization of the KPEs does not require sophisticated computer hardware or software package(s) and can be achieved in a very short time (on the order of seconds).

The second perspective is the question of the ease with which SEISPT could be used with current-generation RTKA systems. It is envisaged that a common set of patient-specific input factors would be utilized for each set of KPEs. These variables would be populated in the patient characterization steps and the only additional information provided to the system would be patient biometric characteristics, such as age, height, and weight. Other patient-specific data are already being recorded to deform the patient-specific anatomical model [44]. Implant component sizing would be done by examining the size of the bone model, as is done in current RTKA surgery practice. Once an implant component size is selected, the corresponding KPE set would be generated, a process that, with the capabilities of current computing systems, would be completed on the order of seconds. Then, the KPE set would be used to generate visual informatics displays that guide final implant component position and orientation. The use of visual informatics displays to communicate KPE results to the surgeon is especially attractive because it allows the surgeon to choose which parameters are given priority. For example, for a particular patient, restoration of proper patellar kinematics may be more important than ligament balancing. In that case, the surgeon can focus on the patellar surface response map to achieve initial positioning of the implant component, and, then, modify the position using the ligament balancing surface response map.

The third perspective is the question of the effect of incorporating a new tool on the surgical workflow when a current-generation RTKA system is used. SEISPT could easily be deployed on any current-generation RTKA system. To illustrate this point, schematic drawings of the surgical workflow for Navio and the proposed surgical workflow for that same system but with SEIST incorporated are presented in Figure 4(a) and Figure 4(b), respectively. As shown (with highlighted items in Figure 4(b)), there are only very small differences between the proposed and existing surgical workflows and, as such, utilization of the workflow plan with SEISPT could be achieved with minimal disruption and, hence, minimal adverse effect on surgery time and cost.

$\underline{\text { Study Limitations }}$

We recognize five limitations of the study. 


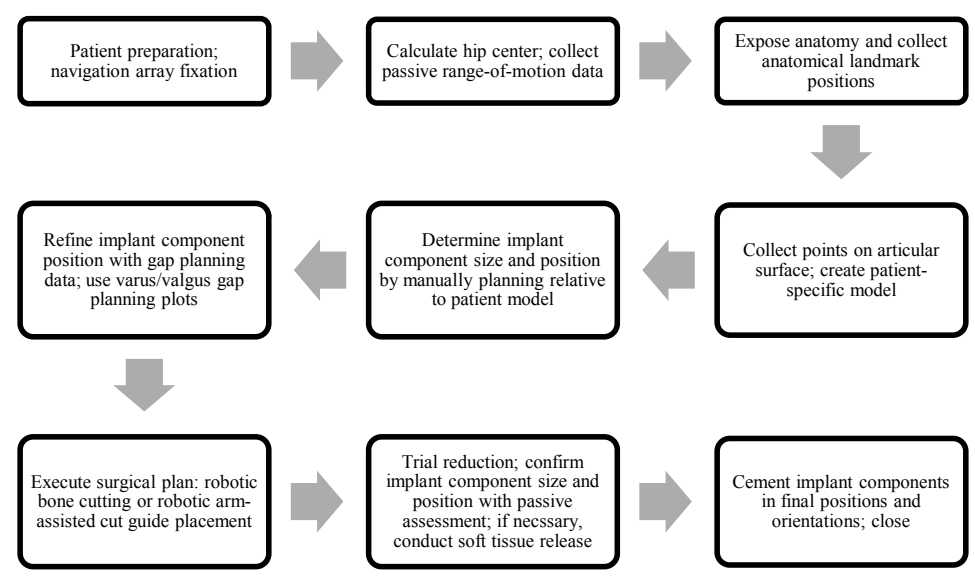

(a)

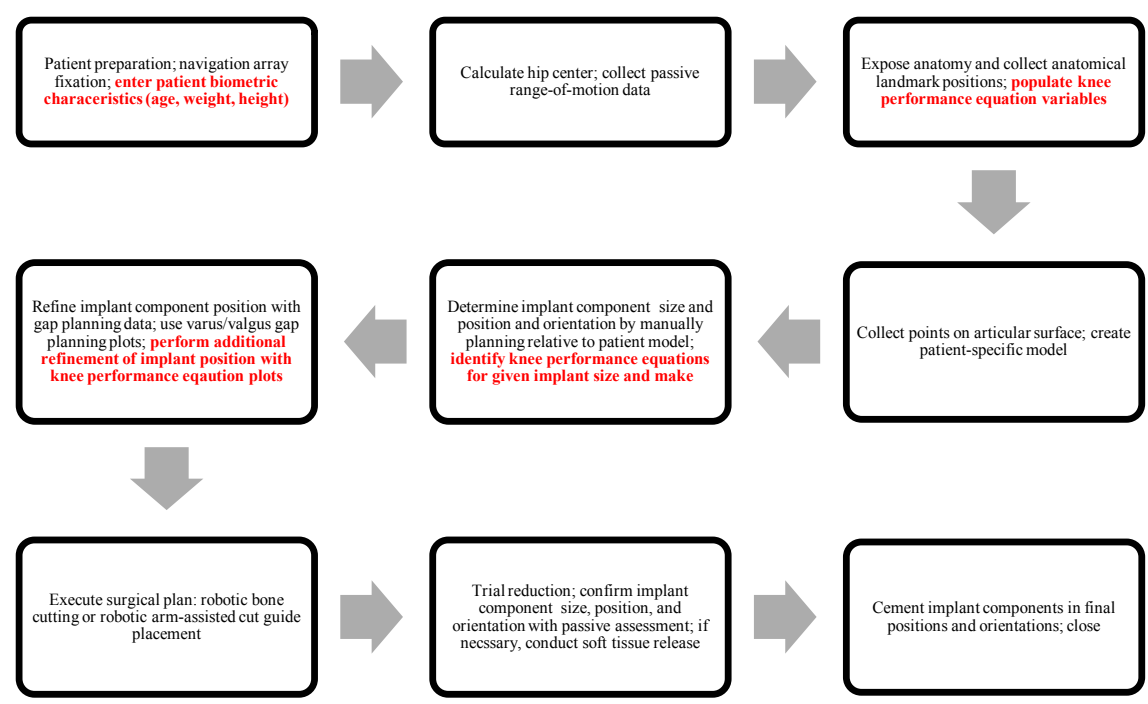

(b)

Figure 4. Schematic diagram of workflow for intraoperative surgical planning used with a current-generation robotic arm-assisted total knee arthroplasty system (Navio ${ }^{\circ}$ [14] (a) and with incorporation of the proposed simulation-enhanced intraoperative surgical planning tool (SEISPT) (b). In (b), steps unique to the incorporation of SEISPT are highlighted in red.

First, there were four shortcomings in some aspects of the musculoskeletal model validation work. One, data from only one subject were used. This is a common shortcoming of studies on validation of musculoskeletal models [45]. Two, the present validation was carried out using one generic multi-body musculoskeletal model, but it is known that for a given functional activity, TFJ contact force predictions show marked variation with the model used [46]. Three, data from two different datasets were used in the validation (OrthoLoad [27] and 2014 SimTK [28] datasets). This was necessitated by the fact that the normal gait data in 2014 SimTK dataset were not available to researchers, such as the present ones, who did not participate in the Grand Challenge Competition. Thus, a loading profile in 2014 SimTK dataset that was similar to that of normal gait profile in another dataset (OrthoLoad) had to be derived. Four, muscle acti- 
vation, which plays a key role in femoro-tibial kinematics [47], was not taken into account in the simulation. It is possible that the third- and fourth-mentioned shortcomings are particularly important in terms of the errors in the computed TFJ force and moment. With regard to this finding, it is worth pointing out that the present study was not a musculoskeletal model simulation work per se; rather, the work was performed to provide results for use in the development of SEISPT.

The second limitation is that in determining the optimal component position and orientation, KPEs that addressed ligament strains were used. Thus, in effect, the surgical plan presented in the present work aims to ensure that, in the postoperative period, the patient does not experience poor proprioception. However, it is understood that determining the optimal implant component position and orientation is a multi-factorial problem that requires a patient-specific solution. In other words, development of a surgical plan must, itself, be multi-factorial. This means that ligament behavior is one of myriad parameters that should be considered, some others being restoration of tibiofemoral alignment and restoration of proper kinematic function. For example, a patient who is at risk for post-operative instability may have a different optimal implant component position than one who is at risk for anterior knee pain.

The third limitation is that in the development of the KPEs, more runs could have been added to the experimental design or a higher-order regression could have been utilized. However, each of these approaches has its shortcoming: longer processing time (former approach) and increased computational burden (latter approach). As the ultimate use of the KPEs was to calculate the optimal position and orientation of implant components, linear equations were used because this approach provided the best balance between long processing time and high computational burden.

The fourth limitation is that the KPE fits could be improved through the use of various transform methodologies, but different transform variants would be required to maximize fits for different KPEs. Thus, the complexity and computational burden associated with improving the fits through data manipulation would be impractical to implement on a large scale.

The fifth limitation is that the results of the proof-of-concept study were compared to those given in biomechanical studies. This was because there are no reports in the clinical literature on the accuracy of component position/orientation when an RTKA system is used.

\section{Conclusions}

Three conclusions were reached, which may be summarized as follows:

First, a simulation-enhanced intraoperative surgical planning tool that may be used in a current-generation RTKA system (SEISPT) was developed. This was achieved by utilizing four steps involving a variety of mathematical techniques, such as design-of-experiments, linear regression, and multi-parameter optimiza- 
tion.

Second, a proof-of-concept study was conducted in which the achieved positions and orientations of the femoral and tibial components of the TKA design implanted in Subject PS (2014 SimTK dataset) were compared to corresponding values obtained through the use of SEISPT. The optimization objectives were set to position and orient the femoral and tibial components to minimize collateral ligament strains while maintaining a physiologically relevant patellofemoral joint contact force. For a given component, the difference in the aforementioned two sets of results was noticeably different from that between achieved and planned results when a current-generation RTKA system (Navio ${ }^{\circ}$ ) was used in a biomechanical test. This suggests that SEISPT, a tool developed using active joint mechanics, may be a plausible alternative to intraoperative surgical planning tools used in current-generation RTKA systems.

Third, three features of SEISPT that point to its feasibility and potential for clinical use are highlighted; in particular, it was shown how incorporation of SEISPT into Navio could be accomplished with minimal disruption and, hence, minimal increase in operative time and cost. In terms of future work, SEISPT should be evaluated in prospective multi-center clinical studies involving Navio as well as other RTKA systems.

\section{Conflicts of Interest}

The authors declare no conflicts of interest regarding the publication of this paper.

\section{References}

[1] Beard, D.J., Davies, L.J., Cook, J.A., Maclennan, G., Price, A., Kent, S., et al. (2019) The Clinical and Cost-Effectiveness of Total versus Partial Knee Replacement in $\mathrm{Pa}$ tients with Medial Compartment Osteoarthritis (TOPKAT): 5-Year Outcomes of a Randomised Controlled Trial. The Lancet, 394, 746-756. https://doi.org/10.1016/S0140-6736(19)31281-4

[2] Bloch, B.V., Palan, J., Shahid, M. and James, P.J. (2020) A New Total Knee Arthroplasty Design Has Significantly Better Early Implant Survivorship than a Previous Gold-Standard Design-A Retrospective Analysis of 1,000 Cases. Journal of Knee Surgery, 33, 152-157. https://doi.org/10.1055/s-0038-1676770

[3] Kahlenberg, C.A., Nwachukwu, B.U., Mclawhorn, A.S., Cross, M.B., Cornell, C.N. and Padgett, D.E. (2018) Patient Satisfaction after Total Knee Replacement: A Systematic Review. HSS Journal, 14, 192-201.

https://doi.org/10.1007/s11420-018-9614-8

[4] Gunaratane, R., Pratt, D.N., Banda, J., Fick, D.P., Khan, R.K.J. and Robertson, B.W. (2017) Patient Dissatisfaction Following Total Knee Arthroplasty: A Systematic Review of the Literature. Journal of Arthroplasty, 32, 3854-3860.

https://doi.org/10.1016/j.arth.2017.07.021

[5] Almeida, G.P.L., De Moura Campos Carvalho e Sliva, A.P., França, F.J.R., Magalhães, M.O., Burke, T.N. and Marques, A.P. (2016) Q-Angle in Patellofemoral Pain: Relationship with Dynamic Knee Valgus, Hip Abductor Torque, Pain and Function. Revista Brasileira de Ortopedia, 51, 181-186. 
https://doi.org/10.1016/j.rbo.2015.05.003

[6] Shervin, D., Pratt, K., Healey, T., Nguyen, S., Mihalko, W.M., El-Othmani, M.M. and Saleh, K.J. (2015) Anterior Knee Pain Following Primary Total Knee Arthroplasty. World Journal of Orthopedics, 6, 795-803.

https://doi.org/10.5312/wjo.v6.i10.795

[7] Innocenti, B., Pianigiani, S., Labey, L., Victor, J. and Bellemans, J. (2011) Contact Forces in Several TKA Designs during Squatting: A Numerical Sensitivity Analysis. Journal of Biomechanics, 44, 1573-1581. https://doi.org/10.1016/j.jbiomech.2011.02.081

[8] Gianola, S.S., Castellini, G., Mascali, M., Vanni, F., Tramacere, I., Banfi, G. and Torneses, D. (2002) Effects of Early Virtual Reality-Based Rehabilitation in Patients with Total Knee Arthroplasty: A Randomized Controlled Trial. Medicine, 99, e19136. https://doi.org/10.1097/MD.0000000000019136

[9] Lonner, J.H. and Fillingham, Y.A. (2018) Pros and Cons: A Balanced View of Robotics in Knee Arthroplasty. Journal of Arthroplasty, 33, 2007-2013.

https://doi.org/10.1016/j.arth.2018.03.056

[10] Kayani, B., Konan, S., Ayuob, A., Onochie, E., Al-Jabri, T. and Haddad, F.S. (2019) Robotic Technology in Total Knee Arthroplasty: A Systematic Review. EFORT Open Reviews, 4, 611-617. https://doi.org/10.1302/2058-5241.4.190022

[11] Antonios, J.K., Korber, S., Sivasundaram, L., Mayfield, C., Kang, H.P., Oakes, D.A. and Heckmann, N.D. (2019) Trends in Computer Navigation and Robotic Assistance for Total Knee Arthroplasty in the United States: An Analysis of Patient and Hospital Factors. Arthroplasty Today, 5, 88-95.

[12] Elbuluk, A.M. and Vigdorchik, J.M. (2019) Total Knee Arthroplasty Technique: NAVIO. In: Lonner, J., Ed., Robotics in Knee and Hip Arthroplasty, Springer, Cham, 145-156. https://doi.org/10.1007/978-3-030-16593-2_15

[13] Shalboch, S., Plaskos, C., Todorov, A., Lawrence, J.M. and Keggi, J.M. (2020) Development of an Active Soft-Tissue Balancing System for Robotic-Assisted Total Knee Arthroplasty. In: Abedin-Nasab, M.H., Ed., Handbook of Robotic and Image-Guided Surgery, Elsevier, Amsterdam, 459-473. https://doi.org/10.1016/B978-0-12-814245-5.00027-X

[14] Mitra, R. and Jaramaz, B. (2020) NAVIO Surgical System-Handheld Robotics. In: Abedin-Nasab, M.H., Ed., Handbook of Robotic and Image-Guided Surgery, Elsevier, Amsterdam, 443-457. https://doi.org/10.1016/B978-0-12-814245-5.00026-8

[15] Cho, K.J., Seon, J.K., Jang, W.Y., Park, C.G. and Song, E.K. (2019) Robotic versus Conventional Primary Total Knee Arthroplasty: Clinical and Radiological Long-Term Results with a Minimum Follow-Up of Ten Years. International Orthopaedics, 43, 1345-1354. https://doi.org/10.1007/s00264-018-4231-1

[16] Ren, Y., Cao, S., Wu, J., Weng, X. and Feng, B. (2019) Efficacy and Reliability of Active Robotic-Assisted Total Knee Arthroplasty Compared with Conventional Total Knee Arthroplasty: A Systematic Review and Meta-Analysis. Postgraduate Medical Journal, 95, 125-133. https://doi.org/10.1136/postgradmedj-2018-136190

[17] Naziri, Q., Cusson, B.C., Chaudhri, M., Shah, N.V. and Sastry, A. (2019) Making the Transition from Traditional to Robotic-Arm Assisted TKA: What to Expect? A Single-Surgeon Comparative-Analysis of the First-40 Consecutive Cases. Journal of Orthopaedics, 16, 364-368. https://doi.org/10.1016/j.jor.2019.03.010

[18] Cool, C.L., Jacofsky, D.J., Seeger, K.A., Sodhi, N. and Mont, M.A. (2019) A 90-Day Episode-of-Care Cost Analysis of Robotic-Arm Assisted Total Knee Arthroplasty. Journal of Comparative Effectiveness Research, 8, 327-336. 
https://doi.org/10.2217/cer-2018-0136

[19] Karunaratne, S., Duan, M., Pappas, E., Fritsch, B., Boyle, R., Gupta, S., Stalley, P., Horsley, M. and Steffens, D. (2019) The Effectiveness of Robotic Hip and Knee Arthroplasty on Patient-Reported Outcomes: A Systematic Review and Meta-Analysis. International Orthopaedics, 43, 1283-1295. https://doi.org/10.1007/s00264-018-4140-3

[20] Jeon, S.W., Kim, K.I. and Song, S.J. (2019) Robot-Assisted Total Knee Arthroplasty Does Not Improve Long-Term Clinical and Radiological Outcomes. Journal of Arthroplasty, 34, 1656-1661. https://doi.org/10.1016/j.arth.2019.04.007

[21] Paszicsnyek, T., Innocenti, B., Bori, E. and Stiegler, C. (2020) Following the Functional Stability Concept during Total Knee Arthroplasty by the Use of a Sensoric Device: First Results with One-Year Follow-Up. Orthopaedic Proceedings, 102-B, Issue Suppl 1, 127.

[22] Siddiqui, A., Smith, T., Mcphilemy, J.J., Ranawat, A.S., Sculo, P.K. and Chen, A.F. (2020) Soft-Tissue Balancing Technology for Total Knee Arthroplasty. JBJS Reviews, 8, e0050. https://doi.org/10.2106/JBJS.RVW.19.00050

[23] Shalhoub, S., Moschetti, W.E., Dabuzhsky, L., Jevsevar, D.S., Keggi, J.M. and Plaskos, C. (2018) Laxity Profiles in the Native and Replaced Knee-Application to Robotic-Assisted Gap-Balancing Total Knee Arthroplasty. Journal of Arthroplasty, 33, 3043-3048. https://doi.org/10.1016/j.arth.2018.05.012

[24] Meere, P.A., Wirekoh, J., Parody, N., Cuevas, R. and Atchabahian, A. (2019) Quantitative Assessment of Tibial Contact Loads from a Straight Leg Raising Test during Total Knee Arthroplasty. Proceedings of the 19th Annual Meeting of the International Society for Computer Assisted Orthopaedic Surgery, New York, 19-22 June 2019.

[25] Dessinger, G., Ta, M., Zeller, I., Nachtrab, J., Sharma, A. and Komistek, R. (2019) In Vivo, Multicenter Assessment of Total Knee Kinematics for Subjects Having Patient-Specific and Traditional Total Knee Arthroplasty. Orthopaedic Proceedings, 101-B, Issue Suppl 4, 64.

[26] Schellenberg, F., Taylor, W.R., Trepczynski, A., List, R., Kutzner, I., Schütz, P., Duda, G.N. and Lorenzetti, S. (2018) Evaluation of the Accuracy of Musculoskeletal Simulation during Squats by Means of Instrumented Knee Prostheses. Medical Engineering \& Physics, 61, 95-99. https://doi.org/10.1016/j.medengphy.2018.09.004

[27] Bergmann, G. (2008) Orthoload. Com. Charite University, Berlin, Germany. http://www.orthoload.com/?page_id-7

[28] Sim, T.K. (2014). https://simtk.org/home/kneeloads

[29] Cheng, H., Obergefell, L. and Rizer, A. (1996) The Development of the GEBOD Program. Transactions of the 1996 15th Southern Biomedical Engineering Conference, Dayton, OH, 29-31 March 1996, 251-254. https://doi.org/10.1109/SBEC.1996.493162

[30] Hast, M.W. and Piazza, S.J. (2013) Dual-Joint Modeling for Estimation of Total Knee Replacement Contact Forces during Locomotion. The Journal of Biomechanical Engineering, 135, Article ID: 021013. https://doi.org/10.1115/1.4023320

[31] Jung, Y., Phan, C.B. and Koo, S. (2015) Intra-Articular Knee Contact Force Estimation during Walking Using force-Reaction Elements and Subject-Specific Joint Model. The Journal of Biomechanical Engineering, 138, Article ID: 021016. https://simtk.org/home/kneeloads

[32] Kirking, B., Krevolin, J., Townsend, C., Colwell, C.W. and D’Lima, D.D. (2006) A Multiaxial Force-Sensing Implantable Tibial Prosthesis. Journal of Biomechanics, 
39, 1744-1751. https://simtk.org/home/kneeloads

[33] Marra, M.A., Vanheule, V., Fluit, R., Koopman, B.H.F.J.M., Rasmussen, J., Verdonschot, N. and Andersen, M.S. (2015) A Subject-Specific Musculoskeletal Modeling Framework to Predict in Vivo Mechanics of Total Knee Arthroplasty. The Journal of Biomechanical Engineering, 137, Article ID: 020904. https://doi.org/10.1115/1.4029258

[34] Evangelista, P.J., Laster, S.K., Lenz, N.M., Sheth, N. and Schwarzkopf, R. (2018) A Computer Model of Mid-Flexion Instability in a Balanced Total Knee Arthroplasty. Journal of Arthroplasty, 33, 265-269. https://simtk.org/home/kneeloads

[35] Kumar, R. and Shahzad, R. (2019) Intraoperative Assessment of Mid-Flexion Instability in Primary Total Knee Replacement. Journal of Pakistan Orthopaedic Association, 30, 93-97.

[36] Saikko, V. (2006) Effect of Contact Pressure on Wear and Friction of Ultra-High Molecular Weight Polyethylene in Multidirectional Sliding. The Proceedings of the Institution of Mechanical Engineers, Part H: Journal of Engineering in Medicine, 220, 723-731. https://simtk.org/home/kneeloads

[37] Churchill, J.L., Khlopas, A., Sultan, A.A., Harwin, S.F. and Mont, M.A. (2018) Gap-Balancing versus Measured Resection Technique in Total Knee Arthroplasty: A Comparison Study. Journal of Knee Surgery, 31, 13-16. https://simtk.org/home/kneeloads

[38] Jaramaz, B., Mitra, R., Nikou, C. and Kung, C. (2018) Technique and Accuracy Assessment of a Novel Image-Free Handheld Robot for Knee Arthroplasty in Bi-Cruciate Retaining Total Knee Replacement. Epic Series in Health Sciences, 2, 98-101. https://simtk.org/home/kneeloads

[39] Hampp, E.L., Chughtai, M., Scholl, L.Y., Sodhi, N., Bhowmik-Stoker, M., Jacofsky, D.J. and Mont, M.A. (2019) Robotic-Arm Assisted Total Knee Arthroplasty Demonstrated Greater Accuracy and Precision to Plan Compared with Manual Techniques. Journal of Knee Surgery, 32, 239-250.

https://simtk.org/home/kneeloads

[40] D’Lima, D.D., Patil, S., Steklov, N., Slamin, J.E. and Colwell, C.W. (2006) Tibial Forces Measured in Vivo after Total Knee Arthroplasty. Journal of Arthroplasty, 21, 255-262. https://simtk.org/home/kneeloads

[41] Lund, M.E., andersen, M.S., De Zee, M. and Rasmussen, J. (2015) Scaling of Musculoskeletal Models from Static and Dynamic Trials. International Biomechanics, 2, 1-11. https://simtk.org/home/kneeloads

[42] Valente, G., Pitto, L., Testi, D., Seth, A., Delp, S.L., Stagni, R., Viceconti, M. and Taddei, F. (2014) Are Subject-Specific Musculoskeletal Models Robust to the Uncertainties in Parameter Identification? PLOS ONE, 9, e112625. https://simtk.org/home/kneeloads

[43] Kapadia, B.H., Berg, R.A., Daley, J.A., Fritz, J., Bhave, A. and Mont, M.A. (2016) Periprosthetic Joint Infection. The Lancet, 387, 386-394.

https://simtk.org/home/kneeloads

[44] Navio Overview. http://www.smith-nephew.com/professional/microsites/navio/naviotechnology/pro duct-overview

[45] Kang, K.-T., Koh, Y.-G., Lee, J.-A. and Chun, H.-J. (2020) Computational Biomechanics of Knee Joint Arthroplasty: A Review. Mechanical Engineering Reviews, 7, Issue 1, Article No. 19-00338.

[46] Wagner, D.W., Stepanyan, V., Shippen, J.M., Demeers, M.S., Gibbons, R.S., An- 
drews, B.J., et al. (2013) Consistency among Musculoskeletal Models: Caveat Utilitor. Annals of Biomedical Engineering, 41, 1787-1799.

https://simtk.org/home/kneeloads

[47] Nejad, Z.I., Khalili, K., Nasab, S.H.H., Schultz, P., Damm, P., Trepczynski, A., Taylor, W.R. and Smith, C.R. (2020) The Capacity of Generic Musculoskeletal Simulations to Predict Knee Joint Loading Using the CAMS-Knee Datasets. Annals of Biomedical Engineering, 48, 1430-1440. 